\title{
Thinking Method of Product Form Design of Bionics Based on Nine Screens
}

\author{
Shigang Wang ${ }^{\mathrm{a}}$, Jie Zhang ${ }^{\mathrm{b}}$, Xifeng Wang ${ }^{\mathrm{c}}$, Fengjuan Wang ${ }^{\mathrm{d}}$ \\ School of Mechatronics Engineering, Qiqihar University, Qiqihar, China

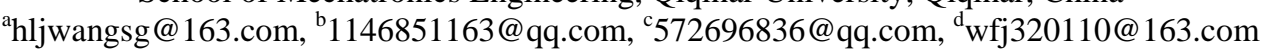

\begin{abstract}
We proposed a thinking method for product form design of bionics to solve the problems of low efficiency and the confused thinking which existing in the current thinking of this field. In the proposed method, we combined the advantages of nine screens method and product form design of bionics, and clear the thinking method of determining bionic object firstly. Then we ascertained the contents of the bionicsnine screens of product form, and separately analyzed the logical relationships among the contents from vertical and horizontal to establish the bionics- nine screens of product form. Combining the thinking method of determining bionic object and the bionics- nine screens of product form, we completely established a thinking mode by showing the thinking flow diagram and explicit method steps. Finally, through the analysis of actual cases, we used this thinking method to propose design schemes for private cars. And the quantity and quality of the schemes prove this method to be systematic, reasonable and efficient.
\end{abstract}

Keywords-nine screens method; product form design; thinking method; bionic object; bionics

\section{INTRODUCTION}

With the rapid development of the society, the industrial products are popularized rapidly, and the product form has become a manifestation of the public utility and aesthetic needs. The improvement of the product form is easier and more effective than the innovation of the product function, but the design of product form is often difficult to make progress because of the traditional thinking [1]. In terms of innovative thinking and design methods, the related knowledge of bionics and TRIZ can improve the design of product form.

The product form design of bionics is to imitate and deform the geometry size, shape and color of the living organisms, and apply it to the new design of product form by means of the corresponding art processing technique [2]. As the comprehensive design of bionics and product form design, its thinking method is various. There are disadvantages of traditional thinking method, such as brainstorming method [3]. Although it can provide a powerful and unconstrained imagine of the design idea, it often leads to low efficiency because of the freedom. And trial-and-error method is more primitive and simple [4], but the work load is heavy and the success rate is low. Above all, there are two reasons. For one thing, the method of determining bionic object according to the design problem is inappropriate. For the other, the thinking and the method are confused during the process of the product form design of bionics.

Nine screens method is one of the important innovative thinking methods in The Theory of Inventive Problem
Solving (TRIZ), which overcomes the inertia of thinking from the view of system and connection. Aiming at solving the contradiction, it solves problems by use of resources [5]. Based on nine screens, in addition to the consideration of the current system, the super system and the subsystem should also be considered; in addition to the current system of the past and the future, super and sub systems of the past and the future should also be considered. It breaks the thinking set from a multilevel utilization of available resources. Compared with other ways of thinking, the nine screens method is more systematic, clearer and more efficient. However, the research and application of the nine screens method is lacking, and less research is used in the product design based on it.

In view of the above problems, this paper analyzes the thinking method of product form design of bionics based on nine screens. It optimizes the determination of bionic object, and proposes the thinking process of product form design of bionics based on nine screens. And through the case of bionic form design of private cars, this thinking method is proved to be systematic, reasonable and efficient.

\section{The Determination Of Bionic OBJect}

In the booming of product form design of bionics, a lot of bionic product designs emerge, which is not considered the function [6]. This blind use of bionic design shortens products' life cycle, wastes resources, and causes other adverse consequences. This is due to the blind of the relation between design problems and the bionic object when designing product form of bionics. And there is no rational understanding to the bionic object and the product itself. In fact, the product form design of bionics is not just thinking about the image. Logic thinking and analysis is also very important. The product form design of bionics based on the nine screens method is needed.

The research object of nine screens method is the system. A system realizes its function by the interaction of multiple subsystems, and the super system a high-level system [7]. In system theory, the product itself composes of various subsystems, with specific features. So corresponding to the super system in nine screens method is the usage environment of products, and the subsystems corresponds to product form. According to the corresponding relation, the three screens of longitudinal direction (super system, systems, subsystems) corresponds to the three factors of product form design (the use of the environment, product, product form), while corresponding to the natural environment, bionic object, bionic form. Fig. 1 shows the relation and correlation between the nine screens method and the product form design of bionics. 


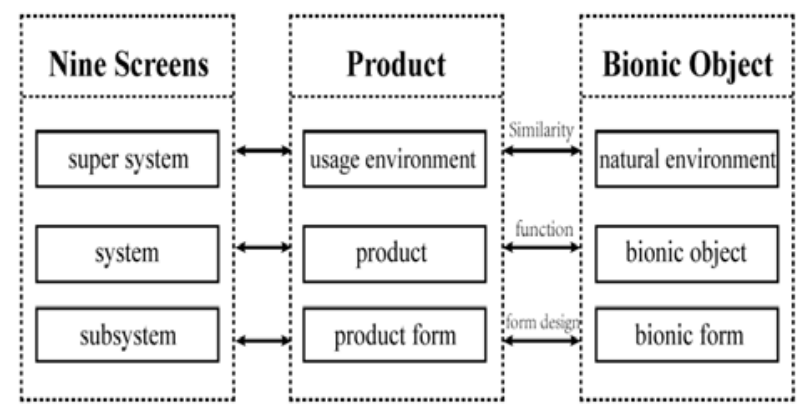

Figure 1. The correlation analysis of system

This correspondence establishes the bridge of nine screens and product form of bionics through learning from each other; and at the same time solves one of the key problems of the product form design of bionics. Then it's easy to search for specific link between bionic products and bionic prototypes, thus to determine the direction of the bionic, even to clear bionic objects. Using the similarity of usage environment of products (super system) and the natural environment to delineate the range of bionic objects and using the function consistency between products and bionic objects to determine the bionic object within the natural environment, and carrying on the art and engineering design in the form of bionic objects in need, so we can easily determine the morphology of the products, serving for product form design.

\section{The CONTENT AND Logical RELATIONSHIP Of ThE BIONIC-NINE SCREENS OF PRODUCT FORM}

\section{A. The Content of the Bionic-nine Screens of Product Form}

The corresponding relationship between form bionics design and nine screens is that the usage environment of products, product and product form correspond to the super system, system and subsystem and horizontal screens correspond the three systems of the past, present, and future. So the content of the nine screens for the product form design of bionics is determined. While the arrow problem of nine screens (the logical relationship between the contents of the nine screens) needs further research. Li Hong [8] considers nine screens should be shown as Fig .2. In fact, when a thinking method is applied to a type of design problems, it should take full advantage of its flexibility in the generality and characteristics to combine organically. Therefore, it should be pruned necessarily based on the nature of nine screens method and product form design of bionics.

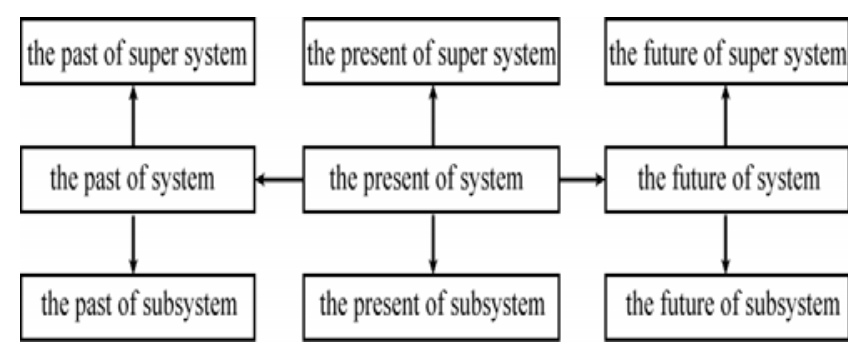

Figure 2. The content of nine screens

\section{B. Analysis of Logical Relationships}

In TRIZ, the solution is put forward according to the problem of product. Aiming at the ideal solution, the innovative product is obtained. Comparing nine screens method and the thinking process of TRIZ, we can clear that nine screens can be divided into two parts, that is, known part (past and present) and the unknown part (future). In order to make clear the logical relationship between the nine screens, we will analyze them in vertical and horizontal.

According to the existing nine screens method, the direction of the arrow is logically related in longitudinal. The process of solving is extrapolating from known to the unknown. The usage environment of products in product form design of bionics is relatively stable, and the product form can be designed after the determination of bionic object (Fig. 1), so these two parameters can be regarded as known. Products in the future are the ultimate goal to be solved, regarded as unknown. Thus the usage environment of products and product form is two important intermediates to restrain product design. So the vertical arrows in the product form design of bionics based on nine screens are shown in Fig .3.
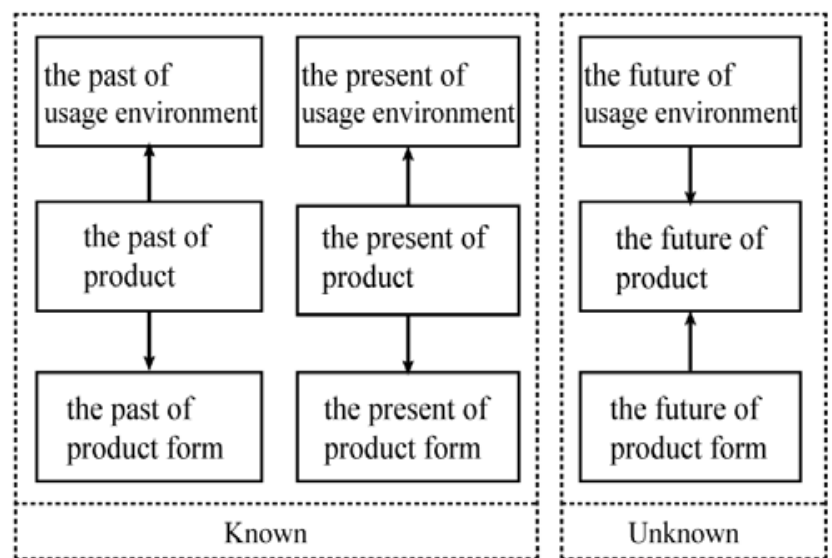

Figure 3. The vertical analysis in the bionics- nine screens of product form

Horizontal screens successively follow the development of product. According to regular pattern of development, the direction of its arrow should be from past to present, then to the future. According to regular pattern of solving problems, the order direction is from known to unknown. In the horizontal, the existing nine screens method did not correlate with the arrows in super system and subsystem level, 
adhering to the principle of "the super system exists with respect to the system". Indeed, if systems in the past and now are functionally different, the super system will be different, so that there is no logical relationship of the direct link and development. But for the product form design of bionics, the form bionic can't damage the function of product [9]. The same usage function of products decides the regular changes of products and the usage environment of products. Listing the different forms of the same product in chronological order, we can summarize the style and design of the same product. So connecting the product forms in the horizontal order by sequential arrows, the thinking can spread along the more reasonable route, and it helps product design well. So the horizontal arrows in the product form design of bionics based on nine screens are shown in Fig .4.

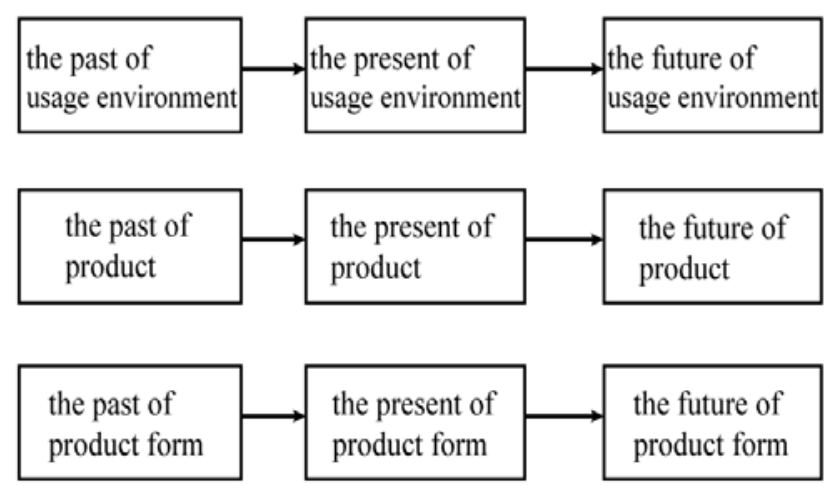

Figure 4. T he horizontal analysis in the bionics- nine screens of product form

\section{The Bionics- nine Screens of Product Form}

In summary, combining the analysis of the horizontal and vertical thinking process, we can draw the complete bionics- nine screens of product form, as shown in Fig .5.

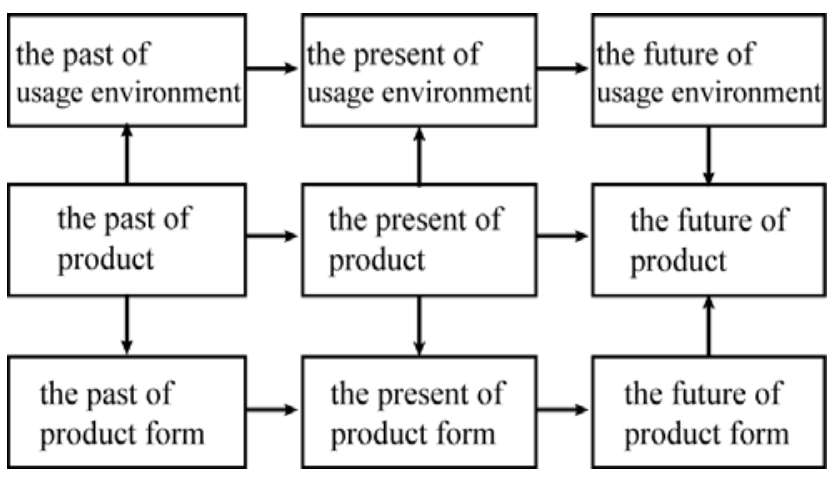

Figure 5. The bionics- nine screens of product form

\section{ThinkIng Method Mode Of Product Form DESIGN OF BIONICS BASED ON Nine SCREENS}

Rational and efficient design thinking is the necessary condition for the successful product form design of bionics. To solve the problems of blind bionic and low efficiency during the design process in existing product form design of bionics, according to the thinking process of "design problem - bionic object - form design", based on the thinking of nine screens method, combining the thinking of nine screens, and through the analysis of nine screens and thinking of form bionic, we summarize up the thinking method mode of product form design of bionics based on nine screens, as shown in Fig .6.

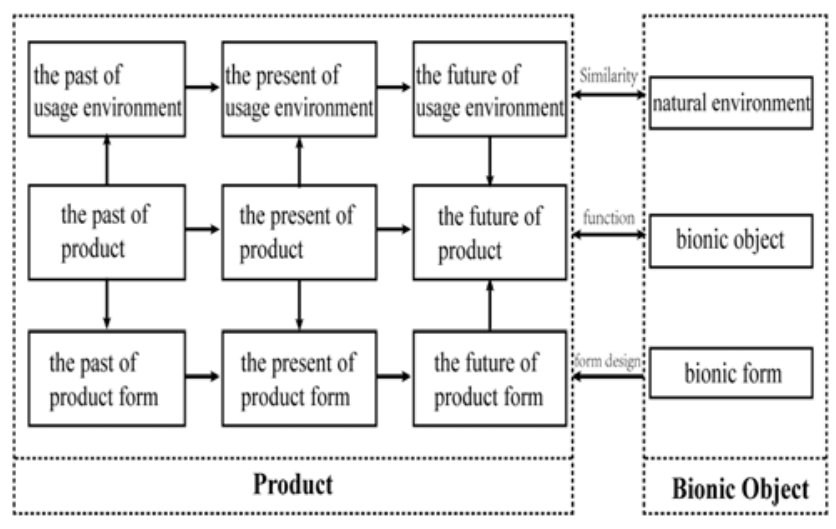

Figure 6. Thinking method mode of product form design of bionics based on nine screens

The thinking process can be briefly as the following steps:

(1) Determine the design problems which need bionic shaping design.

(2) List the nine screens of product form bionics: Base on the design problems, to function as the guidance, list out known screen and resources.

(3) By the known screen to deduce unknown screen: According to the Logical relationship of nine screens of product form bionics, the known resources, and technology evolution law, set using the environment of the future, predict the product and its' form law of development.

(4) Determine the bionic object and its form: Establish the relationship between product function and its usage environment, looking for a relationship which has the similarity relationship with it in the natural environment. To function as the guidance and looking for bionic object and screening; Analogy to the demand between products and product form on the Function, extract the bionic form from the bionic object.

(5) Bionic form design: the bionic form extract from the bionic object need to be abstract, and combined with the development rule and constraints of system, super system, subsystem come from the nine screens of product form bionics, used of the available resources for product form design.

\section{AnAlysis Of ACtual CASES}

Along with the rapid development of science and manufacturing, demands for industrial products in practical and aesthetic is creasing day by day. The form bionic, as a design direction with development potential very much, is widely accepted by designer, but the thinking of the form bionics design is often neglected. In fact, the thinking of 
product form design of bionic based on nine screen method have the advantage of more systematic and efficient.

Take the private cars' form design as an example. Transportation become quick and convenient day by day, soaring demand for private cars, and its' form change and development by leaps and bounds. The development of aerodynamics and streamline style, greatly promoted the development of private cars' form bionics design [10], but on the other hand, constrained the expansion of thinking. So in early stage of development, fish, birds, fins and wings were in the majority as bionic form. A private car of Volkswagen, although the original intention is the natural wind tunnel model, but it's more like a "beetle" and became a classic (as shown in Fig .7). In fact, if no accidental coincidence, through the analysis of nine screens of product form bionics thinking method, can get similar design imagine efficiently.

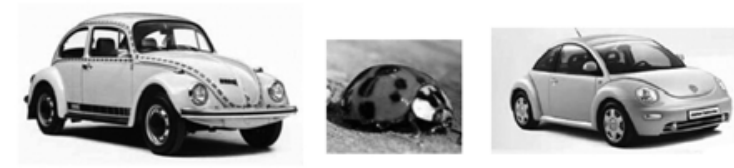

Figure 7. Volkswagen Beetle Car

First, make sure design problems. The form of private cars changed from original geometry to streamline, obviously effective on overcome the air resistance, but, it also caused the low space utilization and protection function abate. So this problem can be described as: if we can reduce the resistance of private cars, at the same time, ensures the space utilization and its' strength

Then list the nine screens of product form bionics, and improve it according to the known screens. Horizontal, according to the development of transportation system and automobile industry, setting the blocked developed roads as the super system, setting the small and medium-sized private cars strengthen the protection function as the system, subsystem is the bionic streamline form. The analysis diagram of nine screens of private cars form bionics is shown in Fig .8.

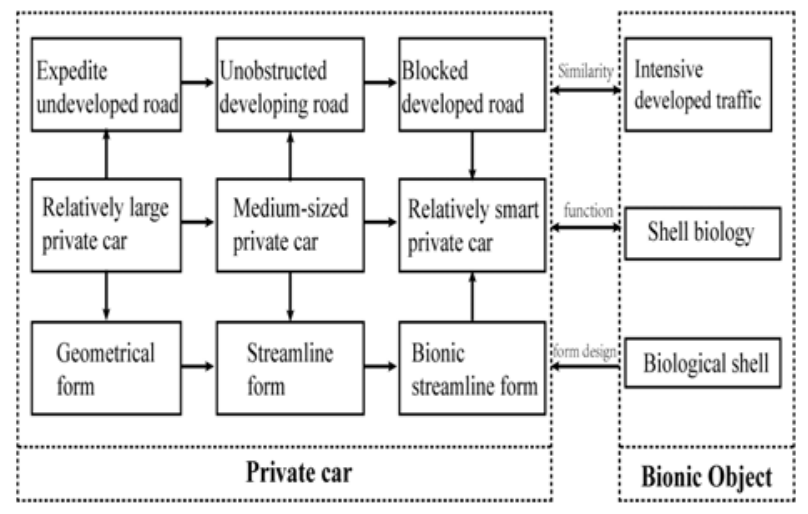

Figure 8. The analysis diagram of bionics- nine screens of private cars' form
Then determine the bionic object and form. The relationship between the protective function of product and operating environment of product makes the small and medium-sized private cars on the blocked developed roads to protect themselves well. By analogy, in the natural environment, shell biological who can protect themselves well in the dense similar, can be identified as bionic object, such as shellfish, golden cicada, dung beetles, snail, dung beetles, turtle, etc. Because of the symbol function of the product, similar to the snail, dung beetles, turtle, etc, which has a negative image object can be abandoned. Then analogy the need of bionic streamline form in subsystem in nine screen, because of its' flexible design space, the appearance structure of shellfish, golden cicada, the beetle, etc, can be used as the bionic form. The last part is bionic form design, as the abstractness of the products has higher aesthetic value, the bionic form need designers use the principle of design aesthetics to abstract itself reasonably.

Of course, the designer's aesthetics design is a great element of success in product form design of bionic, but after the above analysis of the thinking process, the bionic form and predictive solutions of private cars, contains both the beetle car design which is successful, and potentially, applicable and unrealized design scheme. And in the next form design, it can be used to analyze the available resources. Visible, with clear thinking processes, can clarify design problem quickly, analyze design factors reasonably, determine the bionic object efficiently, extract the bionic form accurately, can provide more efficient and reasonable service for product form design of bionic.

\section{CONCLUSIONS}

This article explores the advantages and disadvantages of nine screens method and product form design of bionic thinking, and the improvement of nine screens was applied to product form design of bionic thinking. Proposes the thinking process of product form design of bionic based on the nine screens method. Use the logical thinking of nine screens to guide the divergent images thinking. Establishes the nine screens of product form bionic figure and thinking process. Lock the bionic object and form based on the link between super system and natural environment, product system function and the bionic object, the product subsystem and the biological form. Provides a more systematic, reasonable and efficient way of thinking for design. The form bionics design of private cars, for example, explores the effectiveness of the thinking method.

The process of thinking is macro model proposed, no detailed method is proposed in the process of bionic form into the product form in this paper. And we need other innovative methods in the theory of TRIZ when deal with these issues, so further research is needed to perfect.

\section{ACKNOWLEDGMENT}

This work was supported by Project of Department of Education of Heilongjiang Province, China (Grant No. 12521597). 


\section{REFERENCES}

[1] D. Guo Nanchu, "Key Techniques Research on Product Bionics Design Shape,” Wuhan University of Technology, No. 9, 2012.

[2] J. Du Jie and Du Yu, "The discussion of product form design of bionics,” China New Technologies and Products, No.20, 2011, pp. 85

[3] J. CHEN Hong-liu, REN Gong-chang, LU Qi, YU Feng-hai, and LI Ping-ping, "Analysis of the Role of TRIZ and Brainstorming on the Promotion of Creative Thinking," Mechanical Design and Manufacturing, vol. 11, 2013, pp. 268-269.

[4] J. HOU Min-feng, "Application of Creative Thought in Industrial Design,” Packing Engineering, vol. 24, 2010, pp. 146-148.

[5] M. CAO Jun-qiang, "The Basic Course and the Innovation Examples of the TRIZ Theory,” Harbin: Heilongjiang Science and Technology Press, 2013.

[6] M. CAI Jiang-yu. WANG Jin-ling, "Bionics Design Study,” Beijing: China Building Industry Press, 2013.

[7] M. Liu Xuntao, Cao He, and Chen Guojing, "Theory and Application of TRIZ,” Beijing: Peking University press, 2011.

[8] J. Li Hong, Yan Huigeng, and Xiao Yu, "Several Points of Nine Screen Method in TRIZ Theory,” Industrial \& Science Tribune, vol. 11, 2012, pp. 93-94.

[9] J. ZHANG Ya-li, "The Research of Product Form Design of Bionics,” Art Science and Technology, vol. 4, 2014, pp. 247.

[10] D. HOU Min-feng, "Research on Bionics Design of Automobile Body Form,” Jilin University, 2012. 